\title{
Robust characterization of microfabricated atomic beams on a six-month time scale
}

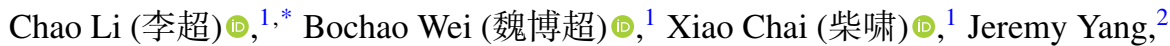 \\ Anosh Daruwalla, ${ }^{2}$ Farrokh Ayazi, ${ }^{2}$ and C. Raman ${ }^{1}{ }^{1}$ \\ ${ }^{1}$ School of Physics, Georgia Institute of Technology, 837 State Street, Atlanta, Georgia 30332, USA \\ ${ }^{2}$ School of Electrical and Computer Engineering, Georgia Institute of Technology, 777 Atlantic Drive NW, Atlanta, Georgia 30332, USA
}

(Received 11 November 2019; accepted 29 April 2020; published 29 May 2020)

\begin{abstract}
Miniature atomic beams can provide new functionalities for atom-based sensing instruments such as atomic clocks and interferometers. We recently demonstrated a planar silicon device for generating well-collimated thermal atomic beams [Nat. Commun. 10, 1831 (2019)]. Here we present fluorescence spectroscopy studies on atomic beams emitted from an array of thin silicon capillaries. These microfabricated rubidium beams work stably over six months at different temperatures above $100^{\circ} \mathrm{C}$. At an oven temperature of $150{ }^{\circ} \mathrm{C}$, the calibrated throughput of the miniature source is $7 \times 10^{11}$ atoms/s/channel with a typical beam brightness of $6 \times 10^{14}$ atoms per second per steradian per unit source area $\left[\mathrm{s}^{-1} \mathrm{sr}^{-1} \mathrm{~mm}^{-2}\right]$. We also present a recipe for evaluating the fluorescence spectra given the Monte Carlo-simulated angular distribution function, even under conditions of strong laser saturation of the probing transition. Monte Carlo simulations together with multilevel master equation calculations fully account for the influence of optical pumping and spatial extension of the Gaussian laser beam. A notable consequence of this work is the agreement between theory and experimental data that has allowed fine details of the angular distribution of the collimator to be resolved over three decades of dynamic range of atomic beam output flux.
\end{abstract}

DOI: 10.1103/PhysRevResearch.2.023239

\section{INTRODUCTION}

Microfabrication techniques have a remarkable opportunity to transform atomic sensors, normally laboratory-scale devices, into portable instruments. Such instruments are urgently needed for precise navigation and timing, electromagnetic field sensing, and gravimetry, all applications where atoms provide a basic reference standard that is traceable to fundamental constants [1-4]. Prototypical examples of atomic platforms currently targeted for these applications are ultracold atoms on a chip and microelectromechanical-based (MEMS-based) microfabricated alkali vapor cells $[5,6]$.

In previous work, we demonstrated a continuous, miniature thermal atomic beam source that propagated along a silicon surface in microchannel arrays defined by photolithography [7]. Such well-collimated sources had never been demonstrated previously at the microscale, to our knowledge. They are new types of chip-scale atomic devices that could find numerous applications, including miniature atom interferometer gyroscopes for inertial guidance [8]. Although the transit time of an atom, and therefore, the gyroscope sensitivity, are reduced compared with free-space setups, other benefits accrue from a chip-scale approach. These include a higher bandwidth of operation and the ability to integrate various sensors on

\footnotetext{
*lichao@ gatech.edu

Published by the American Physical Society under the terms of the Creative Commons Attribution 4.0 International license. Further distribution of this work must maintain attribution to the author(s) and the published article's title, journal citation, and DOI.
}

chip together for multiple axis measurements that are typically challenging to achieve in free-space atom interferometer setups [9]. These sources might also be useful for studies of atom-surface interactions $[10,11]$ including the intriguing possibility to use atoms to directly detect the mechanical motion of MEMS-fabricated structures [12]. Moreover, these atomic beam sources have significantly reduced Doppler broadening compared with vapor cells (a factor of 40 at $100{ }^{\circ} \mathrm{C}$ operation). Single-photon optical excitation on low-lying transitions can therefore be nearly natural linewidth limited, with little to no parasitic absorption from the usual Doppler wings in vapor cells. The absence of ground-state atoms moving at random velocities in the sample might also benefit experiments on miniature Rydberg atom sensors [13], where collisions with such atoms contribute to decoherence.

In this work we have studied the long-term behavior of these sources to prove their robustness and suitability for applications outside of a laboratory. We did so by taking long-term data on the output flux and spectral width using a sample that was made to operate under a 24-hour-per-day, 7-day-per-week regimen for over 4200 hours, a total period of nearly 6 months. Over this time the collimator properties were superbly stable, with no channel clogging observed. This test demonstrates the usefulness and reliability of these microfabricated atomic sources.

Since these sources are necessarily miniature, one needs to study the atomic beam close to the source itself, where the atoms' spatial and velocity distributions are mixed together, i.e., a regime where $z_{0}<w_{x} / \theta_{1 / 2}$. Here, $z_{0}$ is the distance between the probe laser and the nozzle exit, $w_{x}$ is the Gaussian radius of the laser beam, and $\theta_{1 / 2}$ is the half-width at halfintensity for the atomic beam (see Fig. 1) [14]. This is in 


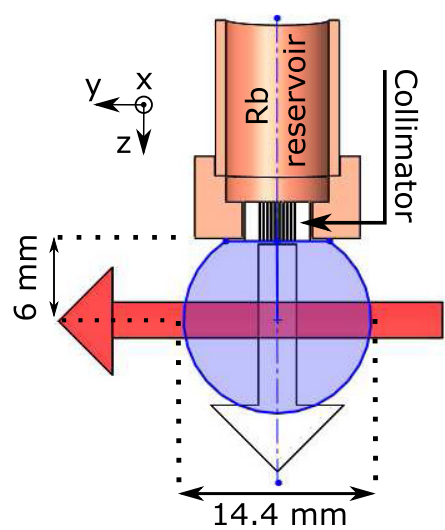

(a)

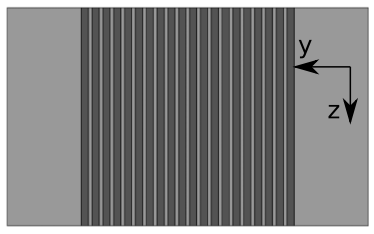

(b)

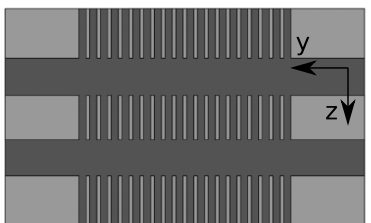

(c)

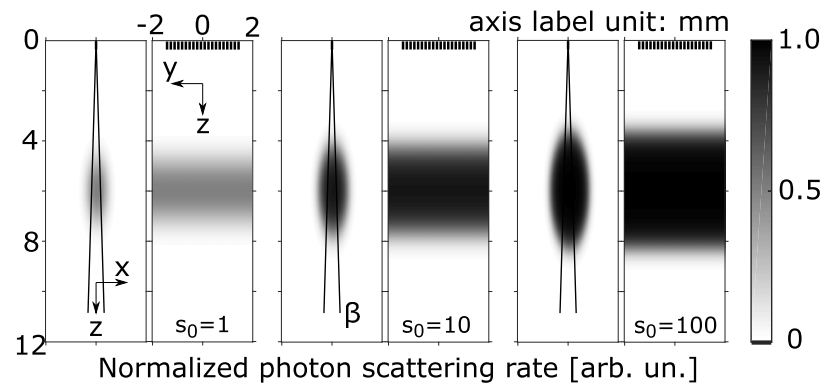

(d)

FIG. 1. Miniature, microfabricated atomic beams probed by fluorescence spectroscopy. (a) A cartoon image showing the close-up view of the front end for a compact atomic beam production and collimation apparatus. A microfabricated silicon device with 20 collimating channels sits inside a slit at the center of the copper head. The red arrow along $+\hat{y}$ represents the laser beam, and the gray arrow along $+\hat{z}$ represents the atomic beams. The shaded region in blue shows the field of view of our fluorescence collecting system. (b) The cartoon top-view of such a collimator [termed an ordinary collimator, in contrast to the cascaded collimator of (c)] before bonding the sealing wafer on top. The chip is $3 \mathrm{~mm} \times 5 \mathrm{~mm}$ in size with $0.5 \mathrm{~mm}$ thickness. For an individual channel, $l / d=30(l=3 \mathrm{~mm}$ and $d=100 \mu \mathrm{m})$. (c) The top view of a three-stage cascaded collimator $(\sim 660 \mu \mathrm{m} /$ stage and $\sim 500 \mu \mathrm{m} /$ gap $)$ with the same overall length and diameter for an individual channel before wafer bonding. (d) Estimated spatial distribution of the normalized resonant photon scattering rate, $s /(1+s)$, in terms of probe saturation parameter $s=I(\vec{r}) / I_{\mathrm{sat}}$, on planes defined by $y=0$ (side view) and $x=0$ (top view), for three different laser intensities corresponding to $s_{\max }\left(\right.$ or $\left.s_{0}\right)=1,10,100$. Each panel is $4 \times 12 \mathrm{~mm}$. The laser beam has Gaussian radius (for $1 / e^{2}$ intensity) $w_{x}=0.5 \mathrm{~mm}$ and $w_{z}=1.4 \mathrm{~mm}$. Bars at the top represent the array of collimator outputs. Two lines forming an angle $\beta$ as a guide depict atoms emitted within the collimator FWHM angle $\beta=2 \theta_{1 / 2}=3.6^{\circ}[14]$.

contrast to typical laboratory-scale atomic beam experiments operating in the far field, where the two distributions can be separated [8,15-17]. In this work, we implement Monte Carlo simulations to construct the spatial and velocity distribution for the chip-scale atomic beam. Multilevel master equation calculation as a companion allows us to compute the fluorescence for noncycling transitions. The predicted fluorescence spectra according to the real experimental configuration agree with our experimental results, thus indicating the validity of our approach.

\section{EXPERIMENTATION}

The fabrication procedure for the chip-scale atomic collimator as well as the fluorescence measurement protocols have been described in detail in the previous work [7]. We briefly review these here, showing the major components of the fluorescence measurement in Figs. 1(a)-1(c). Thermal atomic beams generated by 20 silicon microcapillaries attached to an effusive oven were probed a few millimeters after the nozzle exit. The microcapillaries consisted of $d=100-\mu \mathrm{m}$-wide, $100-\mu$ m-deep channels etched into a silicon wafer, with a typical channel length of $3 \mathrm{~mm}$. A top view of these channels is shown in Figs. 1(b) and 1(c). An unetched capping wafer was bonded to the top of these channels to form a sealed structure, with rubidium entering each channel as a vapor on one end and exiting the other as an atomic beam. In all, 20 atomic beams were generated, each spaced by $150-\mu \mathrm{m}$ center-to-center distance. They copropagated along the $+\hat{z}$ direction on the $y-O-z$ plane as defined in Fig. 1(a).

An external cavity diode laser at $780 \mathrm{~nm}$ used as a fluorescence probe is scanned over a $1-\mathrm{GHz}$ range across the ${ }^{87} \mathrm{Rb} \mathrm{D}_{2} F=2$ to $F^{\prime}=3$ transition at a rate of $5 \mathrm{~Hz}$. The laser beam is propagating perpendicular to the traveling direction of the atom beam along the $+\hat{y}$ direction centered at $z_{0} \simeq 6 \mathrm{~mm}$, and it is linearly polarized along the $z$ axis to maximize the collected fluorescence. Fluorescence emitted from the volume shown in Fig. 1(a) at the intersection of the laser and atomic beams is collected through two 2-inch lenses (not shown) located $\simeq 3$ inches above this volume. Light is collected onto a photodiode, and the photocurrent is amplified by a lownoise current amplifier. Because of the transverse Doppler effect, the fluorescence collected at different laser detunings is sensitive to $v_{y}$, the velocity component along the laser beam. Collimators tailor the velocity components that are perpendicular to the $z$ axis, and thus their projected transverse velocity $v_{y}$. Therefore, better collimation means a narrower measured fluorescence spectrum. Part of the laser output is injected into a rubidium vapor cell at room temperature for saturated absorption spectroscopy, calibrating its operating frequency and assisting the scan control.

We performed two sets of experiments for the two different types of collimators shown in Figs. 1(b) and 1(c). The cascaded collimator [Fig. 1(c)] was reported to generate atomic beams with a 40 times larger peaking factor, the ratio of center-line intensity to the total flow rate, compared to the ordinary-type single-stage collimator [Fig. 1(b)] [7,18]. 


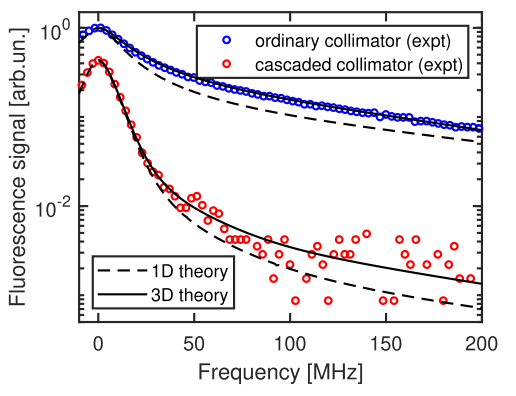

(a)

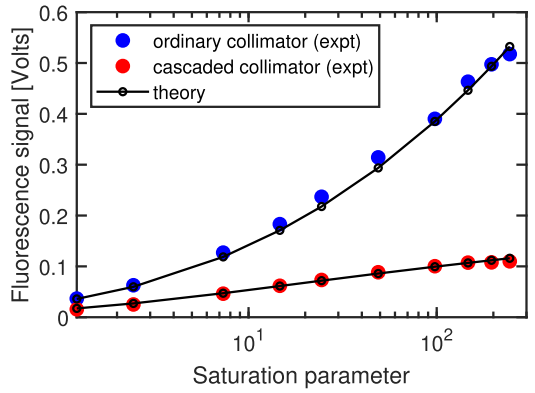

(b)

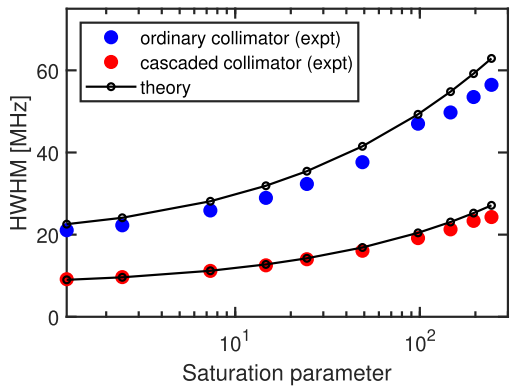

(c)

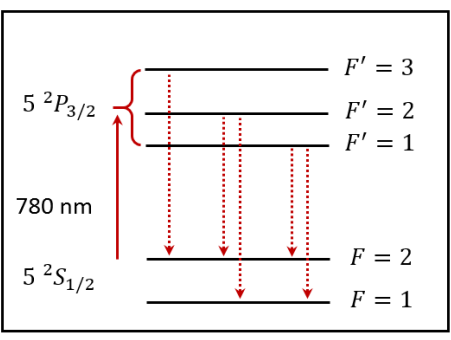

${ }^{87} \mathrm{Rb} D_{2}$ transition hyperfine structure

(d)

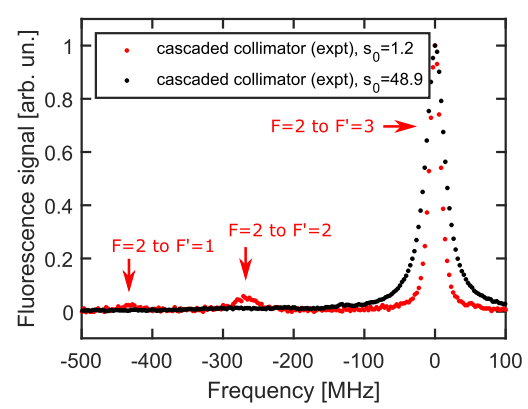

(e)

FIG. 2. Measuring the angular distribution function of atomic collimators. (a) Fluorescence spectra vs laser scan frequency at a saturation parameter of $s_{0}=1.2$ in a $\log$ scale. Blue (red) circles are experimental data for the ordinary (cascaded) collimator. 1D (3D) theory is shown in dashed (solid) lines. (b) Measured peak height of the fluorescence spectra vs $s_{0}$. Blue (red) dots are experimental data for the ordinary (cascaded) collimator. Corresponding solid lines show the theory. (c) Measured HWHM of the fluorescence spectra vs $s_{0}$. Corresponding solid lines show the theory. Error bars for the experimental data in (b) and (c) are smaller than the sizes of the blue and red dots. (d) Relevant ${ }^{87} \mathrm{Rb} \mathrm{D}_{2}$ optical hyperfine transitions. $F$ labels the ground states. $F^{\prime}$ labels the excited states. The solid curve with an arrow labels the laser excitation. Dotted lines with arrows label the spontaneous emission allowed by the selection rules. (e) Red (black) dots show the measured fluorescence spectra for the cascaded collimator at $s_{0}=1.2\left(s_{0}=48.9\right)$. Arrows indicate the hyperfine transitions.

This is because the two gaps efficiently release atoms whose trajectory deviates from the central axis, thus behaving like a filter as discussed in Ref. [7]. For each collimator, we recorded fluorescence spectra over ten different laser intensities adjusted by varying the probe laser power. All other experimental parameters, such as laser beam alignment, beam width, and propagation direction, were kept identical from one collimator to another, so that the spectra could be directly compared with each other.

\section{RESULTS}

\section{A. Experimental spectra}

In general, laser spectroscopy is most accurate at low illumination (low saturation parameter $s=I / I_{\text {sat }}$ ) in order for the measurement to be minimally perturbative of the system under study. Here $I_{\text {sat }}$ is the saturation intensity for the particular optical transition being probed. However, fluorescence detection benefits from a higher probe intensity in order to overcome background noise caused by environmental light and detector or electronic noise sources. Therefore, in practice one needs to work at an appreciable value of $s$, especially when trying to infer the beam characteristics quickly without averaging over long time periods. In our case we would like to resolve the fluorescence spectrum at large detunings to probe the atomic emission at large angles to the collimator axis. Therefore, we have developed a method to extract this velocity distribution even at finite values of $s$ where saturation cannot be ignored.

Figure 2(a) shows our main experimental result. We show experimentally measured spectra over a 200-MHz range of detunings that agree very precisely with a full three-dimensional numerical calculation according to Eq. (10). By contrast, the simple one-dimensional theory used in our earlier work [7] shows clear deviations at the level of $30 \%$ that are particularly pronounced for the ordinary collimator of Fig. 1(b). While the best agreement occurs, as expected, for low saturation parameters, we have also systematically investigated in Figs. 2(b) and 2(c) the influence of laser intensity on the fluorescence spectra up to saturation parameter $s_{0}=I_{0} / I_{\text {sat }}=250$. Here $I_{0}$ is the laser peak intensity, and $I_{\mathrm{sat}}=3.05 \mathrm{~mW} / \mathrm{cm}^{2}$ is for linearly polarized light coupling the ${ }^{87} \mathrm{Rb} \mathrm{D}_{2} F=2$ to $F^{\prime}=3$ transition $[19,20]$. Good agreement is found throughout the range of parameters explored.

In Fig. 2(a) we have plotted data on the blue side of the atomic resonance, as it avoids contamination from the $F=2$ to $F^{\prime}=2,1$ transitions occurring at -267 and $-424 \mathrm{MHz}$, respectively, as well as contributions from the ${ }^{85} \mathrm{Rb}$ isotope's hyperfine transitions [19]. Therefore we can regard our atoms as a pure two-level system for the $F=2$ to $F^{\prime}=3$ transition, with the spectral wings truly representative of the atoms' transverse Doppler velocity distribution within $0 \sim 200 \mathrm{MHz}$, a range roughly corresponding to atoms emitted into an angle of $0 \sim 30^{\circ}$ with respect to the $z$ axis. The far wings are not yet dominated by the Lorentzian in this range due to the long 
tail of the transverse velocity distribution, especially for the ordinary collimator. The transition natural linewidth is $\Gamma=$ $2 \pi \times 6.1 \mathrm{MHz}$, but other mechanisms contribute to broadening the spectra [19]. These include power broadening, transit-time broadening, Doppler broadening, Zeeman broadening, collision broadening, as well as the finite laser linewidth [21]. The power broadening dominates over these other mechanisms for the parameters of our experiment, and scales as $\Gamma \sqrt{1+s}$ with respect to the saturation parameter. For example, at $100{ }^{\circ} \mathrm{C}$, the transit time for atoms going though the laser beam is estimated to be $2 w_{z} / \bar{v} \approx 9 \mu \mathrm{s}$, contributing a transittime broadening of about $0.1 \mathrm{MHz}$. The nozzle heater coil wrapped onto the copper head [shown in Fig. 1(a)] has a DC current running through it that generates a magnetic field with a strength less than 1 Gauss, corresponding to less than $\sim 0.23 \mathrm{MHz}$ line broadening [19]. No significant Zeeman broadening is also verified by momentarily turning off the nozzle heater and monitoring whether the spectra become narrower. Since the mean free path is larger than the probe beam diameter, the collision broadening can be neglected because of the low on-axis atom number density theoretically estimated to be $\sim 6 \times 10^{7}$ atoms $/ \mathrm{cm}^{3} 6 \mathrm{~mm}$ away from the nozzle exit ( $9 \mathrm{~mm}$ away from the reservoir) at $T=100{ }^{\circ} \mathrm{C}$ [22]. The laser linewidth is $<1 \mathrm{MHz}$. Hereafter, we discuss mainly power broadening and the Doppler effect, since all other mechanisms together contribute a total broadening at the $\sim 1 \mathrm{MHz}$ level that can be neglected.

It is interesting to compare the different theoretical curves shown in Fig. 2(a). Our one-dimensional (1D) theory assumes that the atom number density only varies with respect to the transverse coordinate $y$ and not the vertical coordinate $x$. It is a useful approach if the laser interrogation occurs far from the source such that $w_{x} / z_{0} \ll \theta_{1 / 2}$. In this limit, one can neglect off-axis atomic trajectories along the $x$ directions, as well as laser intensity variations. In reality, however, our probe is close to the source, as seen in Figs. 1(a) and 1(d), and one must account for the full three-dimensional nature of both the laser beam profile as well as atomic trajectories through the beam. For our data, we see that the $1 \mathrm{D}$ theory accurately reproduces the spectral width but not the wings of the data. Atomic trajectories at large angles to the central axis contribute most to these wings.

To gain a further qualitative understanding by considering the optical analog of free molecular flow passing through a tube [18], we can divide the population into two components: atoms traveling inside $\theta_{1 / 2}$ (beam component) and outside $\theta_{1 / 2}$ (diffuse component). The half width at half maximum (HWHM) of the transverse velocity distribution $P\left(v_{y}\right) d v_{y}$ for the beam component can be reasonably estimated by $\bar{v} \theta_{1 / 2}$. The diffuse component contributes primarily to the spectral wings of the data, i.e., $\left|v_{y}\right|>\bar{v} \theta_{1 / 2}$. It mainly originates from atomic scattering within the tube $[14,18,23]$.

We can think of the laser beam as a bundle of light rays traveling along $y$ with different $x$ and $z$ coordinates. All rays that lie in the $x=0$ plane intersect both the beam component and the diffuse component. However, rays in the $z=z_{0}$ plane with $|x|>z_{0} \theta_{1 / 2}$ will interact only with the diffuse component. Thus, the latter will measure a broader spectra. We quantify this result further in Fig. 4(c). The final spectrum is a sum over rays with different coordinates, weighted by the contributions of different atomic densities, trajectories, and laser intensities. For our simulations, we assumed that the atomic velocity distribution emitted into any direction obeys the Maxwell-Boltzmann distribution (in traditional atomic physics texts this is known as setting the deformation function to $1[14])$.

Armed with this theoretical approach, we apply it to a variety of different saturation parameters used in the experiment. In Figs. 2(b) and 2(c), we show the peak height and HWHM vs saturation parameter $s_{0}$ of values swept from 1 to 250 for two sets of measured fluorescence spectra for the ordinary collimator and the cascaded collimator, respectively. Our theory can successfully reproduce the measured peak fluorescence intensity as well as the HWHM of the measured spectra. This agreement was achieved without any adjustable parameters for Fig. 2(c), assuming the known saturation intensity of $I_{\text {sat }}=3.05 \mathrm{~mW} / \mathrm{cm}^{2}$ for linearly polarized light coupling the ${ }^{87} \mathrm{Rb} \mathrm{D}_{2} F=2$ to $F^{\prime}=3$ transition. As for Fig. 2(b), only one fitting parameter is introduced as a correction factor $f_{c}$ of the theoretical throughput $\dot{N}$ (discussed in Sec. IV B). What appears to be peculiar about this result is that there is no apparent saturation effect at all. The measured curves in Fig. 2(b) continue to increase with laser intensity, although the rate of growth at high values of $s_{0}$ is slower for the cascaded collimator than for the ordinary collimator. This apparently counterintuitive behavior is a consequence of the laser interrogation region being close to the source, a condition not satisfied in most experiments, where a clear saturation can be observed [15]. As the laser intensity increases, the scattering rate in the center of the beam saturates, while in the wings, it can continue to grow. The ordinary collimator contains a diffuse component that encounters the laser beam wings. By contrast, the cascaded collimator does not contain this diffuse component and therefore shows a less pronounced rate of growth. This effect is well captured by our three-dimensional theory, although the measured spectral HWHM was slightly lower than predicted, as will be discussed later.

\section{B. Optical pumping}

In addition to the saturation behavior explored earlier for the cycling transition, we also deduced and quantified the influence of high probe laser intensity on optical pumping for the other two hyperfine transitions occurring on the red side of the fluorescence spectra [see Figs. 2(d) and 2(e)]. Quantifying optical pumping was necessary to fully understand the role of these other lines in the longitudinal velocity distribution measurements that are discussed later in Fig. 3(e). Optical pumping is also relevant for population leakage during atomic beam slowing and cooling [24], as well as enhancing the signal-to-noise ratio for beam-based atomic clocks or gyroscopes $[8,25,26]$.

Figure 2(e) shows broader spectra inclusive of all relevant hyperfine transitions. To understand the line strengths one must consider various timescales in the collection of the spectra. First, there is the atomic transit time of $9 \mu \mathrm{s}$, which is long enough for atomic state redistribution to occur during the interaction with the probe light. If the probe is on resonance, moreover, it will stay within the resonance width of $6 \mathrm{MHz}$ for $500 \mu \mathrm{s}$, much longer than the transit time, as the laser scan rate 


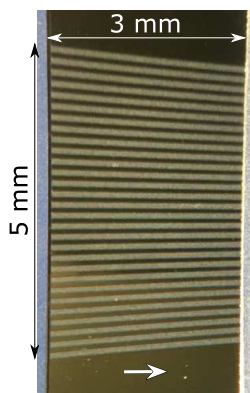

(a)

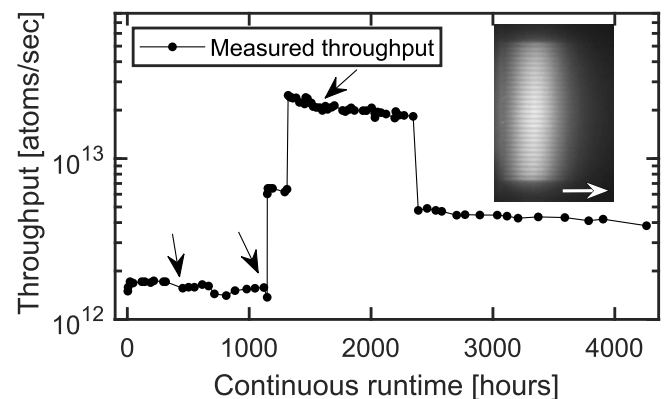

(b)

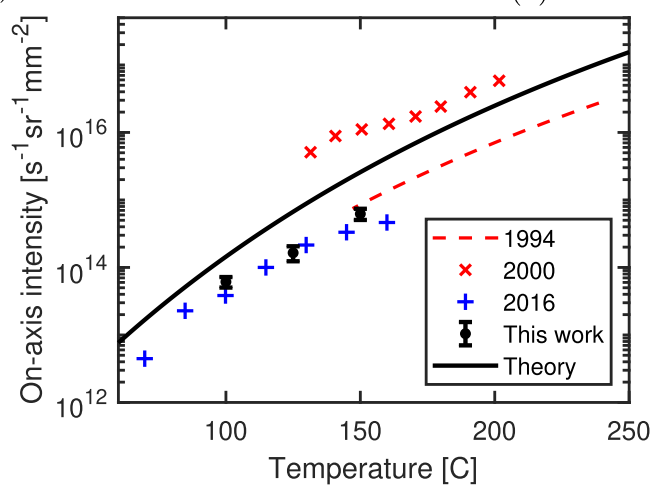

(d)

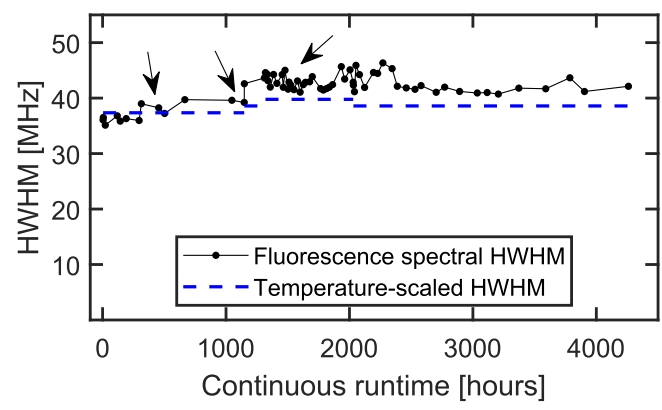

(c)

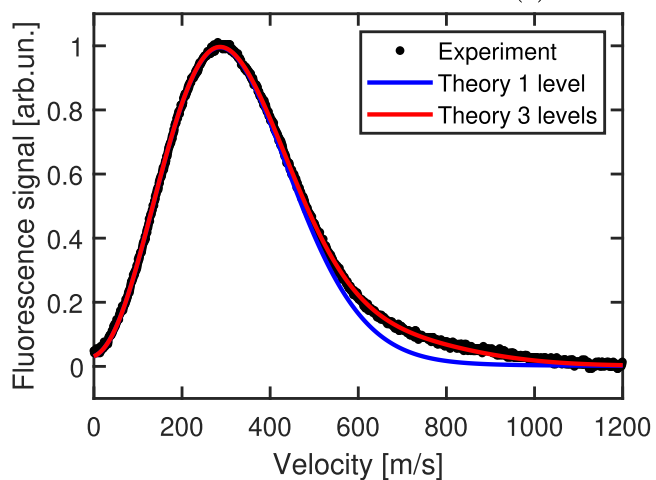

(e)

FIG. 3. Atomic beam production continuous test. (a) Optical top view of the microfabricated collimator without the capping wafer. (b) Experimentally measured total throughput (error bars of $17 \%$ not shown) vs run time. (c) Black dots connected by lines show the measured HWHM of the fluorescence spectra (error bars of $7 \%$ not shown). The blue dashed lines show the benchmarks that are scaled to $125^{\circ} \mathrm{C}$ and $150{ }^{\circ} \mathrm{C}$ using the averaged value at $100^{\circ} \mathrm{C}$. (d) Our measured atomic flux has been converted to the on-axis intensity [ $\mathrm{s}^{-1} \mathrm{sr}^{-1}$ ] normalized by its source area $\left[\mathrm{s}^{-1} \mathrm{sr}^{-1} \mathrm{~mm}^{-2}\right]$, shown in black dots with error bars. (e) Measured longitudinal velocity distribution for the cascaded collimator at a temperature of $150{ }^{\circ} \mathrm{C}$. Also shown are computed fluorescence spectra for a single Maxwell-Boltzmann distribution and three overlapping distributions, as described in the text. Figures 3(b)-3(d) are data for the collimator shown in Fig. 3(a). Figure 3(e) is data for a collimator of the type shown in Fig. 1(c).

is only $12 \mathrm{kHz} / \mu$ s. During transit, then, atoms can be optically pumped to the dark $F=1$ ground state, where no further fluorescence occurs, via the transitions $F=2 \rightarrow 1,2$ (due to the $\Delta F=0, \pm 1$ selection rules, no optical pumping occurs on the $F=2 \rightarrow 3$ transition). Thus as the probe laser intensity increases, more pumping occurs, and the line strengths of $F=2 \rightarrow 1,2$ decrease relative to $2 \rightarrow 3$, as seen in Fig. 2(e). For our experimental parameters, optical pumping occurs within a few $\mu \mathrm{s}$, as determined by multilevel master equation simulations discussed in Sec. IV C. These simulations also quantitatively predict the observed line strengths.

\section{Long-term collimator output}

While the collimating device is relatively simple, for practical applications such as clocks and other precision instruments, it needs to be run for years without servicing. Traditional beam clocks have had to deal with the issue of nozzle clogging [22,27]. For miniature, chip-scale collimators it is an open question as to what extent clogging occurs in practice. To address this issue, we performed limited lifetime tests under continuous operation for a period of 6 months at various fixed temperatures. Our tests could both (i) probe the overall flux emitted from the source using a photodetector to capture the fluorescence as well as (ii) measure the output of every single individual microchannel using a CCD camera focused directly on the output of the chip. A camera image as an inset in Fig. 3(b) for the atomic beam fluorescence right after the channel exit indicates all 29 channels are open (no clogging). The white arrow on the inset marks the propagation direction of the atomic beams. Three black arrows mark the time at 454, 1120, and 1510 hours in Figs. 3(b) and Fig. 3(c) when we took camera images for the collimator output. By contrast, a channel-by-channel test of atomic beam behavior is not easily accomplished using three-dimensional capillary array sources [22,28]. For our tests, the $\mathrm{Rb}$ oven body was kept at a constant temperature, while the chip temperature was between 10 and $30^{\circ} \mathrm{C}$ higher. Temperature differences across the oven body of $5^{\circ} \mathrm{C}, 10^{\circ} \mathrm{C}$, and $13^{\circ} \mathrm{C}$ correspond to oven operating temperatures of $100{ }^{\circ} \mathrm{C}, 125^{\circ} \mathrm{C}$, and $150{ }^{\circ} \mathrm{C}$.

For the throughput measurement, we probed the atomic beam $60 \mathrm{~mm}$ downstream from the chip after the beam had passed through a stainless-steel plate with a 9-mm hole that was $36 \mathrm{~mm}$ away from the chip. This aperture assisted in preventing $\mathrm{Rb}$ vapor from accumulating in the probe region and contaminating the measurement. The vacuum was maintained at $\simeq 6 \times 10^{-6}$ Torr throughout the experiment. As before, the laser probe was perpendicular (misalignment $\leqslant 17 \mathrm{mrad}$ ) to the atomic beam, with a similar fluorescence collection, detection, and amplification setup. The optical collection efficiency 
was $1.2 \%$ known to $12 \%$ of accuracy, and the power of the probe laser was $0.25 \mathrm{~mW}$, with a saturation parameter $I / I_{\text {sat }}=3.8 \pm 0.2$. Here, $I$ is the average intensity that is half the peak intensity of the Gaussian laser beam. We calibrate the total throughput using the measured peak fluorescence and the velocity distribution by following the procedure similar to Ref. [29]. Details are summarized in the Appendix.

The total throughput is shown in Fig. 3(b). We set the oven temperature to $100^{\circ} \mathrm{C}$ at the beginning, $125^{\circ} \mathrm{C}$ at $1150 \mathrm{~h}$, $150{ }^{\circ} \mathrm{C}$ at $1320 \mathrm{~h}$, and $125^{\circ} \mathrm{C}$ again at $2390 \mathrm{~h}$. At the time of writing, this oven had run for over $4200 \mathrm{~h}$ and none of the microchannels showed any sign of clogging. It can be noticed in Fig. 3(b) that the throughput is decaying slowly, which might result from the migrating of $\mathrm{Rb}$ inside the oven from hotter spots to colder spots. The coating of $\mathrm{Rb}$ on the vacuum windows over time, and the drift of laser alignment may also contribute to this decaying effect. Since the flux is around an order of magnitude higher at $150^{\circ}$ and three times higher at $125^{\circ}$, our results implies a continuous operation time at $100^{\circ}$ of over 19000 hours, more than 2 years, without failure. This test proved that our microfabricated atomic beams are reliable and robust at different temperatures and can have a very long lifespan.

While the total flux might be constant, an open question is whether the collimator angular divergence might experience long-term drifts. To address this, in Fig. 3(c) we have plotted the measured HWHM of the measured fluorescence spectrum over the same, roughly 6-month, time period. It is seen to be quite constant over this time period, ruling out long-term degradations or changes in the MEMS fabricated structures due to interaction with rubidium [30,31].

At $100{ }^{\circ} \mathrm{C}$, the mean value of the measured HWHM in Fig. 3(c) is about $37 \mathrm{MHz}$, somewhat larger than for the collimators used in Fig. 1, which were 9 and $21 \mathrm{MHz}$. This is due to the fact that we used a focusing-type collimator with 29 channels pointing to a focal point that is $2 \mathrm{~cm}$ away from the nozzle exit, with a maximum angle of $0.1 \mathrm{rad}$. Therefore, many of the channels contributed a longitudinal component to the spectrum.

For these data, over such a long time period, a background rubidium vapor pressure slowly builds up, which contributes a Doppler pedestal of $\sim 260 \mathrm{MHz}$ HWHM at room temperature [21]. The amount of this vapor is both temperature and time dependent and therefore not easily subtracted from the main atomic beam signal. Nonetheless, we could clearly identify the pedestal after the temperature was raised to $150{ }^{\circ} \mathrm{C}$, where it was largest. The data shown after $1320 \mathrm{~h}$ have the pedestal subtracted. For reference, we have plotted dashed lines that show the $\sqrt{T}$ variation of the most probable atomic velocity, where $T$ is the temperature in Kelvins. Our data after $1320 \mathrm{~h}$ are approximately $10 \%$ higher than this line, indicating that collisions may not be negligible for our conditions, but their influence, if any, is relatively stable over time.

In Fig. 3(d) we have compared our collimating device to a sodium beam source [32] and two other rubidium beam sources [33-35]. Our on-axis atomic beam intensity is about three times smaller compared to the theory for the transparent regime [14] but aligns with the extrapolation from Ref. [32] and data from Ref. [34]. We suspect the calibration in Ref. [33] overestimates the on-axis intensity by a factor of about $3[33,35,36]$. Further enhancement of the atomic beam brightness can be done using transverse laser cooling $[37,38]$. Procedures for computing the on-axis beam intensity are documented in the Appendix.

Apart from the transverse widths measured in Fig. 3(c), we have also investigated in detail the longitudinal velocity distribution on a separate chip employing the cascaded collimator geometry that has been shown in Fig. 1(c). Figure 3(e) shows the measured data. It was collected by intersecting a probe laser at an angle of $\theta_{D}=48^{\circ}$ (this value was determined by a fit using experimentally measured constraints between $45^{\circ}$ and $50^{\circ}$ ) to the atomic beam and recording the emitted fluorescence as the laser frequency was scanned. The Doppler velocity was calibrated as $v=\delta \nu \lambda / \cos \theta_{D}$, where $\delta \nu$ is the frequency detuning from the strong $2 \rightarrow 3$ transition. Due to the sign of the Doppler shift, the $2 \rightarrow 2,1$ lines were shifted to the high-velocity tail of the $2 \rightarrow 3$ transition.

The data show excellent agreement with simple MaxwellBoltzmann statistics. Theoretical spectra were generated using both a single strong line as well as all three overlapping lines $2 \rightarrow 3,2,1$. Good agreement was obtained with just the single strong line, and including the additional lines resolves the small discrepancy visible in Fig. 3(e). The spectra were generated by setting the line temperature to the nozzle temperature of $150{ }^{\circ} \mathrm{C}$ and convolving with the power-broadened natural linewidth of $20 \mathrm{MHz}$. The relative line strengths are the only adjustable parameters, and these were determined by a fit to the data to be $1,0.05$, and 0.03 for $2 \rightarrow 3,2$, and 1 , respectively. These numbers are consistent with the line strengths observed in Fig. 2(e).

These data demonstrate that the collimator longitudinal velocity distribution is not perturbed by collisions even at temperatures of $150{ }^{\circ} \mathrm{C}$, where the output flux is $\sim 7 \times 10^{11}$ atoms/s/channel. For the cascaded collimator, the density in the channels drops rapidly (by more than a factor of 10) after the first gap. Thus only the first collimator section of length $l=0.7 \mathrm{~mm}$ plays a role in collisions. From Table I, the mean free path at the collimator entrance is $\lambda=0.5 \mathrm{~mm}$, yielding an average Knudsen number of $\mathrm{Kn}=$ $2 \lambda / l=1.4$, where the factor of 2 accounts for the linear drop in density across each of the collimator sections. Since $\mathrm{Kn}>1$, we do not expect collisions to be significant for this collimator. For the ordinary collimator the density varies smoothly until the exit. Using $l=3 \mathrm{~mm}$ in the above formula yields $\mathrm{Kn} \simeq 0.3$, indicating that a transitional flow may be approaching.

\section{THEORY}

Our theoretical calculations combined (i) Monte Carlo simulations of the atomic flux in the molecular flow regime based upon the actual experimental geometry of the collimator imported from a CAD model and (ii) an atom-by-atom computation of the fluorescence spectrum based upon each atom's interaction with the laser beam along its particular trajectory using the geometry defined in Fig. 1(a). For the latter, we used a master equation simulation to compute the population of each excited hyperfine level to deduce the fluorescence rate. Details of these calculations are provided in the subsequent sections. 
TABLE I. A table shows the rubidium vapor pressure $P$ and estimated mean free path $\lambda$ vs different temperatures $T$. The vapor pressure is computed based on fits from Ref. [19]. Fits in Ref. [42] give $\sim 20 \%$ smaller vapor pressure for this temperature range.

\begin{tabular}{lcc}
\hline \hline$T[\mathrm{C}]$ & $P[\mathrm{~Pa}]$ & $\lambda[\mathrm{mm}]$ \\
\hline 50 & $6.5 \times 10^{-4}$ & $3.4 \times 10^{2}$ \\
70 & $3.5 \times 10^{-3}$ & $6.8 \times 10^{1}$ \\
90 & $1.6 \times 10^{-2}$ & $1.6 \times 10^{1}$ \\
110 & $5.9 \times 10^{-2}$ & 4.5 \\
130 & $2.0 \times 10^{-1}$ & 1.4 \\
150 & $5.9 \times 10^{-1}$ & $5.0 \times 10^{-1}$ \\
\hline \hline
\end{tabular}

\section{A. Monte Carlo simulations}

We used MOLFLOW+, a test-particle Monte Carlo simulator dealing with molecular flow, to simulate atomic trajectories that pass through the laser beam [40]. We assume that the collimators operate largely in the transparent regime [14], where the mean free path $\lambda$ for atomic collisions is much larger than the length of an individual collimating channel $l$. For our parameters, $l=3 \mathrm{~mm}$, while $\lambda$ can be estimated using the following equation:

$$
\lambda=\frac{k_{B} T}{\sqrt{2} \pi d_{a}^{2} P},
$$

where $P$ is the pressure and $d_{a}$ the atomic diameter. The effective atomic diameter $d_{a}$ can be estimated from the Rb-Rb collision cross section $\sigma=1397 \AA^{2}$ (i.e., $\sigma=\pi d_{a}^{2}$ ) [41]. This condition begins to break down at temperatures of $120^{\circ} \mathrm{C}$ $\left(150^{\circ} \mathrm{C}\right)$ for the single-stage (cascaded) collimators, as shown in Table I. However, uncertainties in the exact temperature and atomic cross section make this an approximate assessment.

The output beam can be fully characterized by an angular distribution function $\kappa f(\theta)$ :

$$
d^{3} \dot{N}=(\dot{N} / \pi) \kappa f(\theta) d^{2} \Omega \Gamma_{\theta}(v) F(v) d v,
$$

where we follow the notation of Ref. [18], with $\kappa f(\theta)$ called the angular distribution function and $F(v)$ the normalized Maxwell-Boltzmann distribution of particle flux. For a single orifice, the angular profile $f(\theta)$, defined as $I(\theta) / I(0)$, equals $\cos \theta$ and therefore $\kappa=1$ [18]. More generally, for a tube, $\kappa \sim 1 / W$ is the "peaking factor" of the collimator, depending inversely on its transmission probability $W=(4 d / 3 l)(1+$ $4 d / 3 l)^{-1}$, where $l, d$ are the tube length and diameter, respectively [18].

One can import CAD geometries for their collimators into MOLFLOW + and specify the inlet surface as an cosine emitter with sticking factor $=1$ mimicking the physics that atoms can return to the source. Sticking factors for all internal surfaces of the collimator are set to be zero, since no $\mathrm{Rb}$ chemical absorption or physical condensation is assumed for crystalline silicon maintained at a temperature that is higher than the rubidium vapor temperature. At the channel exit, another facet with sticking factor $=1$ captures all atoms emitted and records their angles while hitting the surface. The atomic gas is considered to be isothermal, neglecting the thermalization over the whole simulation when atoms enter the microchannels kept at higher temperature than the oven. After releasing enough test particles, since the statistical error varies with $1 / \sqrt{N}$ [43], the angular distribution function can be recovered using the following relation without distinguishing their velocity distribution:

$$
C\left(\theta_{n}\right) \propto \int_{\theta_{i}}^{\theta_{f}} \int_{0}^{2 \pi} \kappa f\left(\theta_{n}\right) d^{2} \Omega,
$$

where

$d^{2} \Omega=\sin \theta d \theta d \phi, \quad \theta_{i}=\theta_{n}-\Delta \theta / 2, \quad \theta_{f}=\theta_{n}+\Delta \theta / 2$,

where $C\left(\theta_{n}\right)$ represents the total number of particles falling into the $n_{t h}$ bin spanned by the differential solid angle integrated over the specified range and $\Delta \theta$ represents the $\theta$ angle sampling step. We did not sample the $\phi$ angle, assuming the angular distribution function has a built-in rotational symmetry with respect to its center line ( $z$ axis), given that the crosssectional shape of collimating tubes has negligible influence on its angular distribution function based on Ref. [39]. $\kappa f(\theta)$ can then be appropriately normalized through

$$
\int_{0}^{\pi / 2} \int_{0}^{2 \pi} \kappa f(\theta) d^{2} \Omega=\pi .
$$

Using MOLFLOW+'s advanced facet parameters, we have finer samplings imposed for small angles $\theta$ to achieve better precision depicting the peaking behavior of the angular distribution function: $\Delta \theta=3.14 \times 10^{-4}$ for $\theta \in\left[0,3.14 \times 10^{-2}\right]$ for the single-stage collimator, while $\Delta \theta=1.57 \times 10^{-4}$ for $\theta \in$ $\left[0,1.57 \times 10^{-4}\right]$ for the cascaded collimator. Another $500 \mathrm{val}-$ ues are equally sampled for $\theta$ angle outside these ranges up to $\pi / 2$, and only one value for $\phi$ angle from 0 to $2 \pi$.

Results for the single-stage collimator and the cascaded collimator are plotted in Fig. 4(a), where the angular distribution functions have been multiplied by its corresponding transmission probability for comparison with the same peak value 1 , noticing the fact that $W \kappa f(\theta=0)=1$. For simple geometries such as the ordinary type of collimators, an analytical expression for the angular distribution function $\kappa f(\theta)$ exists; hence we plot the results given by MOLFLOW+ together with the analytical expression. The good agreement between the two confirms the validity of MOLFLOW + , which can be applied to any nontrivial structures, e.g., the cascaded collimators.

At the center axis of the Gaussian laser beam as shown in Fig. 1(a), we then set up two square targets facing towards the collimator output. The two targets are sitting $6 \mathrm{~mm}$ away from the nozzle exit and detecting the number of hits per unit area per unit time. The impingement rate distributions for two different types of collimators are shown in Fig. 4(b). More than $99 \%$ of atoms are actually emitted into the halos for the traditional-type single-stage collimator. This can smear out the fine spectra features, contaminate nanostructures or microcavities [16,44-46], and reduce transparency of optical accesses. In contrast, the cascaded collimator with the identical physical size can produce better collimated atomic beams with 40 times fewer atoms emitted into large angles while maintaining the same axial beam intensity of $n A \bar{v} /(4 \pi)$ [22]. 


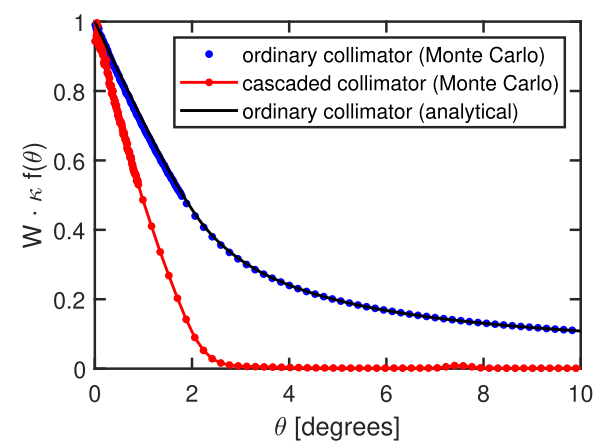

(a)

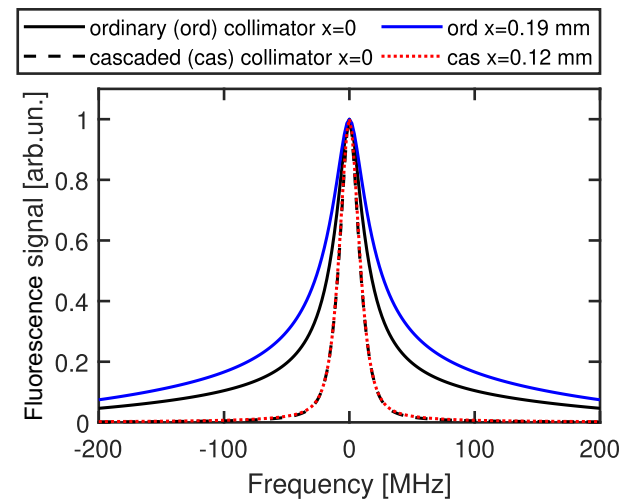

(c)

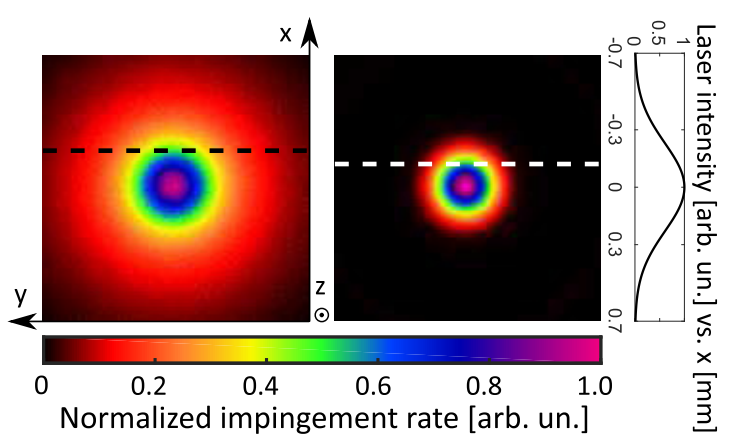

(b)

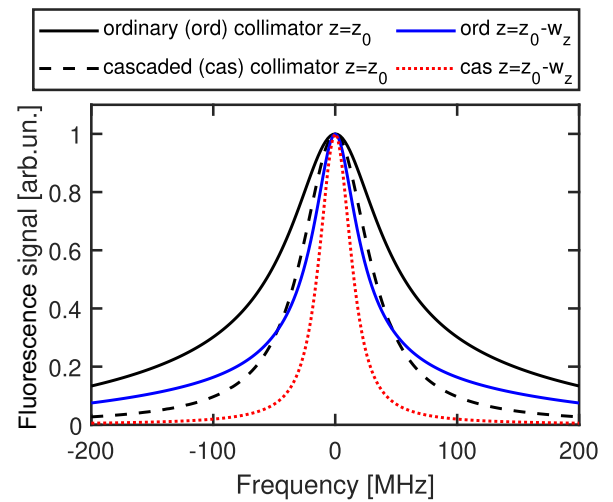

(d)

FIG. 4. Monte Carlo simulation of atomic beams allows full characterization of atomic fluorescence spectra. (a) Angular distribution functions $\kappa f(\theta)$ multiplied by their corresponding transmission probabilities (or Clausing factors) $W$. Blue and red are for Monte Carlo simulations. The black solid line represents the analytical result available only for the ordinary collimator [18,39]. (b) The predicted impingement rate distribution for an ordinary collimator and a cascaded collimator. Each target is $1.4 \times 1.4 \mathrm{~mm}$ in size with $10 \times 10 \mu \mathrm{m}$ pixels. Impingement rates are respectively normalized to their peak values for these two targets. For reference, the laser beam intensity profile in the target plane is plotted to the right side. Circles sharing the same impingement rate form contours. The dashed line is tangent to a contour, where the impingement rate has dropped to a half. The computed fluorescence spectra for the ${ }^{87} \mathrm{Rb}_{2} \mathrm{D}_{2}=2$ to $F^{\prime}=3$ transition show the atomic and laser beam inhomogeneous effects. (c) At low saturation parameter $s_{0}=1$, the computed spectrum is broader at $x=0.19$ for the ordinary collimator because of the diffuse component of the atomic beam. This component is missing in the cascaded collimator, hence the spectra are very similar at $x=0$ and $x=0.12$. The black (white) dotted lines in (b) indicate $x=0.19(0.12$ ). (d) At high saturation parameter $s_{0}=100$, the computed spectrum is narrower in the Gaussian wings compared with the center. Shown are spectra along different single lines on the $y-O-z$ plane, where the laser intensity is at its peak value $\left(z=z_{0}\right)$ or at $1 / e^{2}$ of its peak value $\left(z=z_{0}-w_{z}\right)$.

\section{B. Fluorescence spectral computation}

To compute the spectrum we need to know the atom number density at a location $\vec{r}$ from the exit of the collimator (refer to Fig. 5, which shows the relevant coordinate system). To compute this, we treat an individual collimator exit face as an effective source of total throughput $\dot{N}$, farfield angular distribution $\kappa f(\theta)$, and source area $A=d^{2}$, where the collimator tube cross section is $d \times d$. We begin by considering a monoenergetic beam of mean velocity $\bar{v}$. With these definitions, $\dot{N} \frac{d A^{\prime}}{A} \frac{\kappa f\left(\theta^{\prime}\right)}{\pi} d \Omega^{\prime}$ is the fractional flux reaching the observation point $\mathrm{P}$ that emanated from a small region of area $d A^{\prime}$ at $\vec{r}^{\prime}$. We equate this to the mean flux passing through the area $d S=\left|\vec{r}-\vec{r}^{\prime}\right|^{2} d \Omega^{\prime}$, which is $n\left(\vec{r}, \vec{r}^{\prime}\right) \times \bar{v} \times d S$, where $n\left(\vec{r}, \vec{r}^{\prime}\right)$ is the contribution to the density at $\vec{r}$ originating from the source at the location $\vec{r}^{\prime}$. Integrating over the source, we get

$$
n(\vec{r})=\int_{A} d A^{\prime} \frac{\dot{N}}{A} \frac{\kappa f\left(\theta^{\prime}\right)}{\pi} \frac{1}{\bar{v}\left|\vec{r}-\vec{r}^{\prime}\right|^{2}} .
$$

At short distances, this integral yields the source density, while at large distances it yields, up to a numerical factor, the angular distribution function. The crossover between near

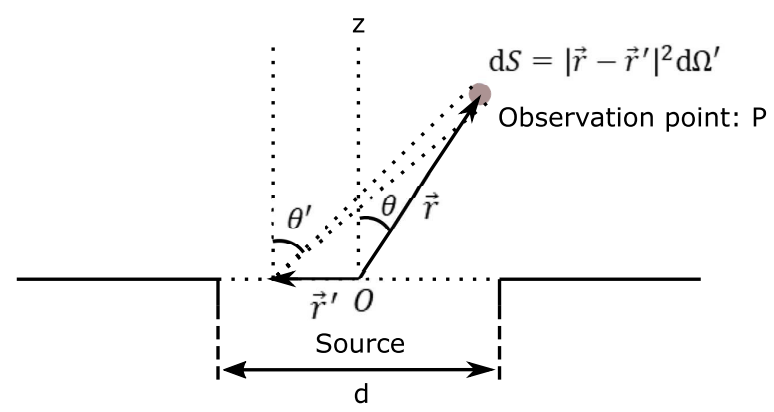

FIG. 5. The coordinate system for computing the atom number density at a certain observation point $\mathrm{P}$. The nozzle exit has an area of $A$. For our microfabricated silicon channel, $A=d^{2}$, and it is about $0.01 \mathrm{~mm}^{2}$. 
TABLE II. A table shows the scaling coefficients as given in Eqs. (8) and (10), used in the calculations for the optical depth and the fluorescence signal. $f_{c}$ is the only fitting parameter, and all others are input parameters.

\begin{tabular}{llc}
\hline \hline Symbol & \multicolumn{1}{c}{ Meaning } & Value \\
\hline$\omega_{0}$ & Laser frequency & $2 \pi \times 384.2 \mathrm{THz}$ \\
$R_{\text {resp }}$ & Photodiode responsivity & $0.6 \mathrm{~A} / \mathrm{W}$ \\
$G$ & Current amplifier gain & $1 \times 10^{8} \mathrm{~V} / \mathrm{A}$ \\
$\eta$ & Photon collecting efficiency & $1.2 \%$ \\
$A_{b}$ & Natural abundance & $27.8 \%$ \\
$f_{p}$ & Fraction of population in $|F=2\rangle$ & $5 / 8$ \\
$N_{t}$ & Number of tubes & 20 \\
$f_{c}$ & Correction factor & 2.4 (cascaded), 2.8 (ordinary) $[7]$ \\
$N$ & Total throughput at $T=100^{\circ} \mathrm{C}$ & $4.2 \times 10^{9} \mathrm{~s}^{-1}$ (cascaded), $1.7 \times 10^{11} \mathrm{~s}^{-1}$ (ordinary) \\
\hline \hline
\end{tabular}

and far fields occurs when $\theta^{\prime} \simeq \theta_{1 / 2}$, the angular width of the source, at a corresponding distance $z=z_{c}=d /\left(2 \tan \theta_{1 / 2}\right)$. Essentially, at $z=z_{c}$, an individual capillary output has diverged by an amount equal to its size. Our measurements are made at $z_{0}=6 \mathrm{~mm}$ from the nozzle exit, which is only somewhat beyond the crossover point, since $z_{c} \simeq 3.8 \mathrm{~mm}$ for our cascaded collimator where $d=100 \mu \mathrm{m}$ and $\theta_{1 / 2}=$ $13 \mathrm{mrad}$. We note, however, that in order to compute the spectrum $n(\vec{r})$ must be integrated over a volume larger than the source dimensions, so no substantial error is incurred by making the far-field approximation. In this case, replacing $1 / \bar{v}$ with $F(v) / v$ [18], where $F(v)$ is the Maxwell-Boltzmann distribution, we get a density of particles at location $\vec{r}$ per unit velocity:

$$
n(\vec{r}, v)=\dot{N} \frac{\kappa f(\theta)}{\pi} \frac{F(v)}{v r^{2}} .
$$

Neglecting atomic absorption of the probe light, we can assume the laser beam intensity varies only with respect to $x$ and $z$, while it does not change much along its propagation axis $y$. We estimate absorption by computing the optical depth (OD) of the sample at the probe location, which we write down as (referring to the coordinate system of Fig. 1)

$$
\mathrm{OD}=2 A_{b} f_{p} N_{t} \int_{0}^{\infty} d y \int_{0}^{\infty} d v n(\vec{r}, v) \sigma\left(\Delta, s_{0}\right),
$$

where the integral $d y$ may be transformed to polar coordinates using $d y=d\left(z_{0} \tan \theta\right), A_{b}$ is the natural abundance of ${ }^{87} \mathrm{Rb}, f_{p}$ is the population fraction for a certain hyperfine level, $N_{t}$ is the total number of microcapillaries (see Table II), $\Delta=k v \sin \theta$, $s_{0}=1.2$, and the on-resonance photon scattering cross section is $\sigma_{0}=\hbar \omega_{0} \Gamma /\left(2 I_{\text {sat }}\right)$. Here, $I_{\text {sat }}=3.05 \mathrm{~mW} / \mathrm{cm}^{2}$ is for linearly polarized light coupling the ${ }^{87} \mathrm{Rb}_{2} F=2$ to $F^{\prime}=3$ transition [19]. We compute the OD via numerical integration, estimating that it is about $1.6 \%(0.6 \%)$ for the ordinary (cascaded) collimator, and therefore we can safely neglect it. The Gaussian laser beam intensity distribution can then be written as

$$
I=I_{0} e^{-2\left(x^{2} / w_{x}^{2}+\left(z-z_{0}\right)^{2} / w_{z}^{2}\right)}
$$

Then the measured output voltage reads

$$
\begin{aligned}
V_{f}(\delta)= & \hbar \omega_{0} R_{\mathrm{resp}} G \eta A_{b} f_{p} N_{t} f_{c}^{-1} \\
& \times \int_{0}^{\infty} d v \int_{B} d V n(\vec{r}, v) R_{s c}[s(\vec{r}), \delta-k v \sin \theta \sin \phi],
\end{aligned}
$$

where $\delta$ is the laser detuning, $\hbar \omega_{0}$ gives the energy per photon, $R_{\text {resp }}$ is the responsivity of the photodiode, $G$ is the current amplifier gain, $\eta$ is the overall photon collecting efficiency, $f_{c}$ is the correction factor [7] for the theoretical total throughput $\dot{N}$, and $B$ is determined by the fluorescence collecting volume. Numerical values of these scaling factors are given in Table II. While seemingly complicated, Eq. (10) can be formulated into a simple linear algebra problem after discretizing the integral (see the Appendix),

$$
\vec{V}_{f}=A\left(s_{0}\right) \kappa \vec{f}
$$

where $A\left(s_{0}\right)$ is a matrix mapping the angular distribution function $\kappa \vec{f}$ into the predicted fluorescence spectra $\vec{V}_{f}$ for a certain saturation parameter $s_{0}$. Theoretical results for Figs. 2(a)-2(c) are readily obtained once $A\left(s_{0}\right)$ is computed for the ten different experimental saturation parameters. One can either predict the angular distribution function using Monte Carlo simulations and then predict the fluorescence spectra treating Eq. (11) as a simple forward problem or measure the fluorescence spectrum and inversely find the angular distribution function $[47,48]$.

In the low-saturation-parameter regime, a broader spectrum can be measured for the single-stage collimator while probing the fluorescence spectrum off the $y-O-z$ plane. We quantitatively verify this by computing the fluorescence spectra using Eq. (10) along the dashed lines and the center lines, and normalize their peak value to be 1 for convenience [see Fig. 4(c)]. That's why the 1D approximation predicts the narrower spectrum compared to the measured data as reported in our previous work [7]. For the cascaded collimator, spectra measured at different $x$ locations are similar, since the number of atoms emitted from tube walls has already been suppressed by 40 times. Atoms passing through the region near the dotted lines for the cascaded collimator mainly come from the beam component rather than the diffuse component.

In the high-saturation-parameter regime, a narrow spectrum can be measured in the Gaussian wings of the laser beam compared to the center when probing the fluorescence spectrum on the $y-O-z$ plane. Following the same procedure above, the spectra are calculated along lines at different $z$ locations [see Fig. 4(d)]. The spectra computed at $z=z_{0}$ is broader than the spectra computed at $z=z_{0}-w_{z}$, where $s=s_{0} / e^{2}$ solely because the power broadening is larger. These spectra share the same nontrivial transverse velocity 
distribution, and thus we can compare the power broadening directly. At the Gaussian wings of the laser beam, the spectral half-width converges towards their intrinsic Doppler broadening, as shown in Fig. 4(c). Analytical estimates are not available for the half-width of the measured fluorescence spectra when the Doppler and power broadening are comparable to each other, considering the atomic or laser beam inhomogeneous effects if probing close to the source.

\section{Master equation simulations}

Figure 2(e) reveals that the red side of the fluorescence spectra manifests a different line strength for the $F=2$ to $F^{\prime}=2,1$ transitions under different laser intensities due to optical pumping effects. A closed-form analytical expression for $R_{s c}$, as required in Eq. (10), is not available for these two transitions, in contrast to the $F=2$ to $F^{\prime}=3$ cycling transition. Therefore, to quantify these optical pumping effects, we need to run master equation simulations to track the dynamical evolution of the excited-state populations contributing to the fluorescence.

The master equation reads as follows $[7,49]$ :

$$
\dot{\rho}(t)=-\frac{i}{\hbar}[H(\vec{r}, t) \rho(t)]+\Gamma\left(\frac{2 J^{\prime}+1}{2 J+1}\right) \sum_{q=-1}^{1} \mathcal{D}\left[\Sigma_{q}\right] \rho(t),
$$

where the first part is for the atom-light interaction and the second part is for the spontaneous decay. At low light level (peak intensity $I_{0}=3.8 \mathrm{~mW} / \mathrm{cm}^{2}$ ) and oven temperature $T=$ $100{ }^{\circ} \mathrm{C}$, an ensemble of atoms is assumed to be traveling with the most probable velocity $v_{\text {beam }}=327 \mathrm{~m} / \mathrm{s}$ for an atomic beam [29,50] going through the light field corresponding to Eq. (9), from $\left(z_{0}-1.5 w_{z}\right)$ to $\left(z_{0}+1.5 w_{z}\right)$, where the light intensity has dropped to a significantly low level. We initialize the density matrix according to the Boltzmann distribution.

Figure 6 shows the population dynamics for both $F^{\prime}=3$ and $F^{\prime}=2$ excitations. Our principal observation is that the $F^{\prime}=3$ excitation can be effectively treated as two level. Figure 6(a) shows that the excited-state population tracks its steady-state value,

$$
P_{e}\left(F^{\prime}=3, \vec{r}(t)\right)=\frac{5}{16} \frac{I(\vec{r}(t)) / I_{\mathrm{sat}}}{1+I(\vec{r}(t)) / I_{\mathrm{sat}}},
$$

where $I(\vec{r}(t))$ is the intensity at the instantaneous location $\vec{r}(t)$ of the atoms. The difference between Eq. (13) and the master equation simulation was negligible. From Eq. (13) we can get the on-resonance photon scattering rate $R_{s c}(\vec{r}(t))=\Gamma P_{e}\left(F^{\prime}=\right.$ $3, \vec{r}(t))$ for atoms at any specific locations. Figure 6(b) shows the populations in $|F=2\rangle$, which can contribute to the collected fluorescence through temporarily occupying $\left|F^{\prime}=2\right\rangle$ and are eventually transferred into the dark state $|F=1\rangle$ that has a $6.8 \mathrm{GHz}$ detuning from the excitation field. An additional dark state is $\left|F=2, m_{F}=0\right\rangle$, for which there is a zero coupling matrix element to $\left|F^{\prime}=2, m_{F}=0\right\rangle[21,51]$. These states become substantially populated within $\sim 5 \mu \mathrm{s}$. The presence of a kink feature in the solid red curve at the beginning stage of the simulation is because the Rabi flopping takes a time about $1 / \Gamma$ to reach steady state due to spontaneous emission, and during that time the system evolves coherently. The integrated number of photons scattered is

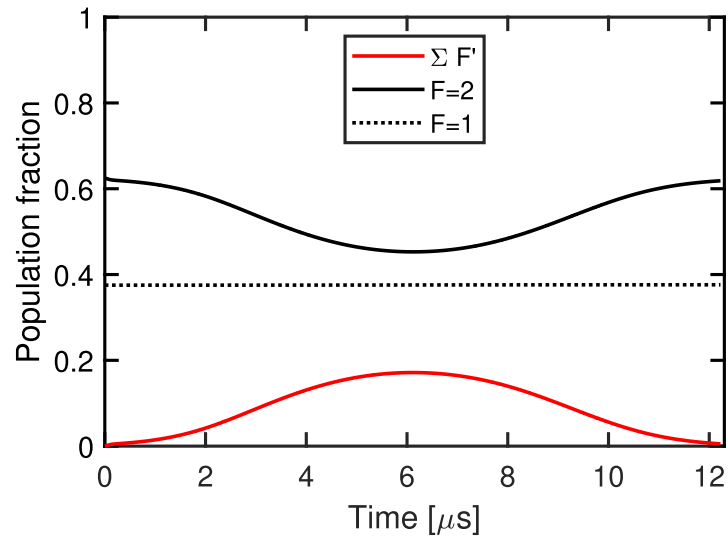

(a)

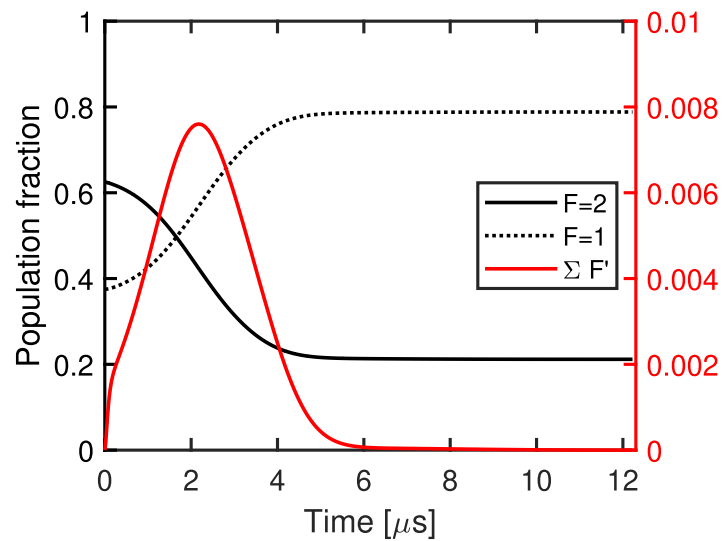

(b)

FIG. 6. Master equation simulations. An ensemble of atoms is traveling through the Gaussian light field. The black curves show the time evolution of the population in individual ground states. The red curve shows the time evolution of the summed population in all excited states $\left(F^{\prime}=0,1,2,3\right)$. The laser frequency is on-resonance with the ${ }^{87} \mathrm{Rb} \mathrm{D}_{2} F=2$ to $F^{\prime}=3$ cycling transition for (a) and is on-resonance with the $F=2$ to $F^{\prime}=2$ noncycling transition for (b), where we have a separate axis on the right for the population in excited states.

computed from the excited-state probabilities and yields good quantitative agreement with the measured spectra in Fig. 2(e). These procedures were previously documented (see Ref. [7]) but not the master equation simulation data of Fig. 6.

\section{CONCLUSION}

In summary, we have developed a Monte Carlo-assisted approach for understanding fluorescence spectra measured under scenarios where laser intensities, atoms' spatial distributions, and velocity distributions are all wound together. The clogging-free continuous operation and the individualchannel addressability identify these on-chip elements as promising candidates, generating well-collimated atomic beams for clocks, interferometry, or atomic-optical device hybridization [25,52-58]. Looking forward, combining Monte Carlo and master equation simulations allows the tracking of atom trajectories and their internal states simultaneously, which is indispensable for customizing the laser manipulation 
of atoms close to or within a chip-scale source itself [24]. The simple recipe phrased in linear algebra for reconstructing the velocity or angular distribution of the atomic beams is important for precision spectroscopy [46,59] or atom scattering experiments [60], and useful for guiding collimator and oven designs $[34,61,62]$.

\section{ACKNOWLEDGMENTS}

We thank Scott Elliott, Nathan Mauldin, and Frank Murdock at the MRDC Machine Shop for manufacturing oven test assemblies. We thank Richard Shafer at the IEN Laser Lab for guidance on the Optec femtosecond laser micromachining system. C.L. acknowledges helpful discussions with Kevin Driscoll, Xin Xing, and Shangguo Zhu.

\section{APPENDIX}

\section{Throughput}

In this section, we summarize the procedure for calibrating the total throughput shown in Fig. 3(b). We estimate the total throughput by following the procedure given in Ref. [29]. We can do a $1 D$ calculation because the atomic flow $\mathcal{Q}$ [atoms/s] is measured at a location $z_{0} \simeq 60 \mathrm{~mm}$ away from the collimator exit (i.e., $z_{0} \gg w_{x} / \theta_{1 / 2}$ ). Let us assume there are $N_{B}$ atoms inside the collection volume defined as $V_{B}=\sigma l_{y}$, where $\sigma=$ $\pi w_{x} w_{z}$, and $w_{x}=0.5 \mathrm{~mm}, w_{z}=1.4 \mathrm{~mm}$ are the Gaussian radius of the laser beam. It will be soon shown that $l_{y}$ will not participate in this calibration. Now the measured atomic flow $\mathcal{Q}$ [atoms/sec] can be written as $\mathcal{Q}=\mathcal{F} \mathcal{S}$, where $\mathcal{S}=2 w_{x} l_{y}$ and $\mathcal{F}$ [atoms $/ \mathrm{sec} / \mathrm{cm}^{2}$ ] represents the flux of atoms at the probe location

$$
\mathcal{F}=\rho v_{\text {beam }},
$$

with $\rho=N_{B} / V_{B}$ and $v_{\text {beam }}=\sqrt{3 k_{B} T / m}\left(k_{B}\right.$ Boltzmann constant, $T$ oven temperature, $m$ rubidium mass) as the most probable longitudinal velocity for effusive atomic beams. Now the measured atomic flow $\mathcal{Q}$ [atoms/s] reads

$$
\mathcal{Q}=N_{B}\left(\frac{\pi w_{z}}{2 v_{\text {beam }}}\right)^{-1},
$$

which can be regarded as $N_{B}$ atoms going through the laser beam with an effective transit time $\pi w_{z} /\left(2 v_{\text {beam }}\right)$. Knowing the photon scattering rate for laser detuning $\delta$ and atoms with transverse velocity $v_{y}$,

$$
R_{s c}\left(\delta-k v_{y}\right)=\frac{\Gamma}{2} \frac{s}{1+s+4\left(\frac{\delta-k v_{y}}{\Gamma}\right)^{2}},
$$

where $s=3.8 \pm 0.2$ is the saturation parameter for the average intensity of the Gaussian laser beam $0, k=2 \pi / \lambda$ is the wave number, and $\Gamma=2 \pi \times 6.1 \mathrm{MHz}$ is the natural linewidth for the corresponding transition, we can relate the number of atoms inside the collection volume $N_{B}$ to the peak voltage $V_{f}(\delta=0)$ of the measured fluorescence spectra in the following way:

$$
V_{f}(\delta=0)=\hbar \omega_{0} R_{\mathrm{resp}} G \eta A_{b} f_{p} N_{B} \int_{-\infty}^{+\infty} d v_{y} R_{s c}\left(k v_{y}\right) P\left(v_{y}\right),
$$

where the numerical values of these scaling factors are given in Table II. In contrast to Ref. [29], where they assumed a normal distribution for transverse velocity, we deconvolve $P\left(v_{y}\right)$ directly from the experimentally measured fluorescence spectra by noticing the fact that the fluorescence spectra $V_{f}(\delta)$ is simply a convolution of the photon scattering rate $R_{s c}\left(\delta-k v_{y}\right)$ and the transverse velocity distribution $P\left(v_{y}\right)$,

$$
V_{f}(\delta) \propto \int_{-\infty}^{+\infty} d v_{y} R_{s c}\left(\delta-k v_{y}\right) P\left(v_{y}\right) .
$$

The process can be done using a Fourier transform routine with Tikhonov regularization [7,63]. To sum up, we (1) deconvolve the transverse velocity distribution $P\left(v_{y}\right)$ using Eq. (A5); (2) find the number of atoms $N_{B}$ within the fluorescence collection volume $V_{B}$ using Eq. (A4); and (3) determine the measured atomic flow $\mathcal{Q}$ [atoms/s] using Eq. (A2). Finally, a test-particle Monte Carlo simulation can tell us a fraction about $\chi \simeq 1.2 \%$ of the total throughput $\dot{N}_{\exp }$ travels through the photon collection volume $V_{B}$ and hence

$$
\dot{N}_{\exp }=\mathcal{Q} / \chi,
$$

which are plotted in Fig. 3(b) for a focusing-type collimator with 29 channels in total.

\section{On-axis intensity}

In this section, we summarize the procedure for calibrating the atomic beam on-axis intensity shown in Fig. 3(d). The onaxis intensity [atoms $/ \mathrm{s} / \mathrm{sr}$ ] of the atomic beam is defined as

$$
I(0)=\lim _{\delta \Omega \rightarrow 0} \frac{\dot{N}(\delta \Omega)}{\delta \Omega},
$$

where $\dot{N}(\delta \Omega)$ is the number of atoms emitted into the differential solid angle $\delta \Omega$ per unit time. For our experimental configuration, only a certain fraction of the measured atomic flow $\mathcal{Q}$, whose transverse velocity is close to zero, of the following

$$
\dot{N}(\delta \Omega)=2 \mathcal{Q} P\left(v_{y}=0\right) \delta v_{y},
$$

is axially emitted into the differential solid angle of

$$
\delta \Omega=\frac{\delta \mathcal{S}}{z_{0}^{2}}=\frac{4\left(\delta v_{y} / v_{\text {beam }}\right) z_{0} w_{x}}{z_{0}^{2}},
$$

where $\delta v_{y}$ is an auxiliary variable specifying a tiny range of transverse velocity and easily gets canceled out in the following. Combining Eqs. (A2), (A8), and (A9) gives an estimation for the experimentally measured on-axis intensity:

$$
I_{\text {exp }}(0)=\frac{N_{B}}{\sigma} v_{\text {beam }}^{2} P\left(v_{y}=0\right) z_{0} .
$$

Although Eq. (A10) has an explicit $z_{0}$ dependence, the number of atoms measured in the fluorescence collection volume $N_{B}$ scales like $z_{0}^{-1}$ because the atom number density scales like $z_{0}^{-2}$ and the fluorescence collection volume $V_{B}$ scales like $z_{0}^{1}$, corresponding to the same range of transverse velocity (divergence angle). Therefore, Eq. (A10) is valid to give an estimation for the on-axis intensity $I_{\exp }(0)$ without actually relying on the fluorescence probing location.

Because the long-term test was done for a focusing array, each individual channel indexed by $i$ has a different contribu- 
tion to the measured on-axis intensity obeying

$$
\sum_{i=-14}^{14} I\left(\theta_{i}\right)=I_{\exp }(0)
$$

where $\theta_{i}=i \times 7.6 \mathrm{mrad}$ with respect to the $z$ axis [see Fig. 3(a)]. Noticing the fact that $I\left(\theta_{i}\right) / I(0)=\kappa f\left(\theta_{i}\right) / \kappa f(0)$ given in Fig. 4 [18], we estimate the on-axis intensity for an individual channel that is parallel to the $z$ axis,

$$
I_{\|}(0)=2.5 I_{\exp }(0) / 29,
$$

which are then normalized by the source area of $0.1 \times 0.1 \mathrm{~mm}^{2}$ and plotted in Fig. 3(d) for this work. An intuitive explanation for this correction is that $P\left(v_{y}=0\right)$ in Eq. (A10) would be larger if the microchannel array was a parallel array, for which a narrower transverse velocity distribution could be measured because all channels would be perpendicular to the probing laser beam.

We have compared $I_{\|}(0)$ to other effusive atomic beam sources in Fig. 3(d) [32-34]. Reference [32] was a sodium source. Figure 2 of Ref. [32] is first fit into a straight line. Then we find the corresponding temperature $T_{R b}$ at which the rubidium vapor pressure $P_{\mathrm{v}}\left(T_{R b}\right)$ [19] equals the sodium vapor pressure $P_{\mathrm{v}}\left(T_{N a}\right)$ [64]. The emission rate per unit solid angle is scaled by a factor of $\bar{v}\left(T_{R b}\right) / \bar{v}\left(T_{N a}\right)$, where $\bar{v}$ represents the average molecular velocity for gas inside the oven [14,18,22]. The beam density $\rho$ plotted in Fig. 2 of Ref. [33] is converted into the on-axis intensity by $I(0)=\rho R^{2} \bar{v}$, where $R=35 \mathrm{~cm}$ as the probe-candlestick distance [22]. The total throughput (flux) $\Phi$ given in Fig. 6 of Ref. [34] is converted into the on-axis intensity by $I(0)=\kappa \Phi / \pi$, where $\kappa$ is a theoretical peaking factor that equals $W^{-1}$, the inverse of the Clausing factor [18]. For comparison, on the same plot in Fig. 3(d), all converted on-axis intensities $I(0)$ from different references have been normalized by their own source area, respectively.

\section{Linear algebra format}

In this section we present a linear algebra recipe for computing the fluorescence spectra shown in Fig. 2. Following Eq. (10), insert

$$
d V=r^{2} \sin \theta d r d \theta d \phi
$$

and

$$
F(v) \propto v^{3} e^{-(v / \alpha)^{2}}
$$

into the expression for the detected fluorescence, and we get

$$
V_{f}(\delta) \propto \int_{0}^{\infty} d v \int d r \int d \phi \int d \theta A\left(v, r, \theta, \phi, \delta, s_{0}\right) \kappa f(\theta),
$$

where we have dropped all constants as coefficients, and $A\left(v, r, \theta, \phi, \delta, s_{0}\right)$ represents

$$
A\left(v, r, \theta, \phi, \delta, s_{0}\right)=\sin \theta R_{s c}\left(r, \theta, \phi, s_{0}, \delta, v\right) v^{2} e^{-(v / \alpha)^{2}}
$$

The field of view of our imaging system defines the collecting volume $B$ and thus determines the integral lower and upper limit for $r, \theta, \phi$ in Eq. (A15). If we define

$$
A\left(\delta, \theta, s_{0}\right)=\int_{0}^{\infty} d v \int d r \int d \phi A\left(v, r, \theta, \phi, \delta, s_{0}\right),
$$

then Eq. (A15) can be rewritten as the following simple expression:

$$
V_{f}(\delta) \propto \int d \theta A\left(\delta, \theta, s_{0}\right) \kappa f(\theta)
$$

The discrete version of Eq. (A18) will be

$$
V_{f}\left(\delta_{i}\right) \propto \sum_{j} \Delta \theta A\left(\delta_{i}, \theta_{j}, s_{0}\right) \kappa f\left(\theta_{j}\right)
$$

Now, the theoretical prediction for the fluorescence spectrum has been completely formulated into a problem of the following type,

$$
\vec{V}_{f}=A\left(s_{0}\right) \kappa \vec{f}
$$

where $\kappa \vec{f}$ represents the discretized angular distribution function $\kappa f(\theta), \vec{V}_{f}$ represents the fluorescence spectrum $V_{f}(\delta)$, and the matrix $A\left(s_{0}\right)$ represents the mapping between the two under different saturation parameters. Interestingly, the matrix $A\left(s_{0}\right)$ does not depend on what type of collimator/angular distribution function we have at all. It intrinsically characterizes the mapping once the laser probe geometry and power are well defined. For absolute fluorescence signal calibration, we absorb previously dropped scaling coefficients back to Eq. (A20) by the end of the computation. Scaling factors defined in Eq. (10) are summarized in Table II.
[1] B. Battelier, B. Barrett, L. Fouché, L. Chichet, L. AntoniMicollier, H. Porte, F. Napolitano, J. Lautier, A. Landragin, and P. Bouyer, Development of compact cold-atom sensors for inertial navigation, in Quantum Optics (International Society for Optics and Photonics, Bellingham, WA, 2016), Vol. 9900, p. 990004.

[2] D. D. McCarthy and P. K. Seidelmann, Time: From Earth Rotation to Atomic Physics (Cambridge University Press, Cambridge, England, 2018).

[3] E. Boto, N. Holmes, J. Leggett, G. Roberts, V. Shah, S. S. Meyer, L. D. Muñoz, K. J. Mullinger, T. M. Tierney,
S. Bestmann et al., Moving magnetoencephalography towards real-world applications with a wearable system, Nature (London) 555, 657 (2018).

[4] Y. Bidel, N. Zahzam, C. Blanchard, A. Bonnin, M. Cadoret, A. Bresson, D. Rouxel, and M. Lequentrec-Lalancette, Absolute marine gravimetry with matter-wave interferometry, Nat. Commun. 9, 627 (2018).

[5] M. Keil, O. Amit, S. Zhou, D. Groswasser, Y. Japha, and R. Folman, Fifteen years of cold matter on the atom chip: Promise, realizations, and prospects, J. Mod. Opt. 63, 1840 (2016). 
[6] J. Kitching, Chip-scale atomic devices, Appl. Phys. Rev. 5, 031302 (2018).

[7] C. Li, X. Chai, B. Wei, J. Yang, A. Daruwalla, F. Ayazi, and C. Raman, Cascaded collimator for atomic beams traveling in planar silicon devices, Nat. Commun. 10, 1831 (2019).

[8] T. L. Gustavson, Precision rotation sensing using atom interferometry, Ph.D. thesis, Stanford University, 2000.

[9] B. Barrett, P. Cheiney, B. Battelier, F. Napolitano, and P. Bouyer, Multidimensional Atom Optics and Interferometry, Phys. Rev. Lett. 122, 043604 (2019).

[10] J. D. Perreault and A. D. Cronin, Observation of Atom Wave Phase Shifts Induced by van der Waals Atom-Surface Interactions, Phys. Rev. Lett. 95, 133201 (2005).

[11] D. Reiche, K. Busch, and F. Intravaia, Nonadditive Enhancement of Nonequilibrium Atom-Surface Interactions, Phys. Rev. Lett. 124, 193603 (2020).

[12] M. Antezza, C. Braggio, G. Carugno, A. Noto, R. Passante, L. Rizzuto, G. Ruoso, and S. Spagnolo, Optomechanical RydbergAtom Excitation via Dynamic Casimir-Polder Coupling, Phys. Rev. Lett. 113, 023601 (2014).

[13] H. Fan, S. Kumar, J. Sedlacek, H. Kübler, S. Karimkashi, and J. P. Shaffer, Atom based RF electric field sensing, J. Phys. B: At. Mol. Opt. Phys. 48, 202001 (2015).

[14] J. Giordmaine and T. Wang, Molecular beam formation by long parallel tubes, J. Appl. Phys. 31, 463 (1960).

[15] M. L. Citron, H. R. Gray, C. W. Gabel, and C. R. Stroud, Jr., Experimental study of power broadening in a two-level atom, Phys. Rev. A 16, 1507 (1977).

[16] D. W. Keith, C. R. Ekstrom, Q. A. Turchette, and D. E. Pritchard, An Interferometer for Atoms, Phys. Rev. Lett. 66, 2693 (1991).

[17] S. Bell, M. Junker, M. Jasperse, L. Turner, Y.-J. Lin, I. Spielman, and R. E. Scholten, A slow atom source using a collimated effusive oven and a single-layer variable pitch coil zeeman slower, Rev. Sci. Instrum. 81, 013105 (2010).

[18] H. C. W. Beijerinck and N. F. Verster, Velocity distribution and angular distribution of molecular beams from multichannel arrays, J. Appl. Phys. 46, 2083 (1975).

[19] D. A. Steck, Rubidium 87 D Line Data, available online https://steck.us/alkalidata (revision 2.2.1, 21 Nov. 2019).

[20] B. Gao, Effects of Zeeman degeneracy on the steady-state properties of an atom interacting with a near-resonant laser field: Analytic results, Phys. Rev. A 48, 2443 (1993).

[21] C. J. Foot, Atomic Physics (Oxford University Press, Oxford, England, 2005), Vol. 7.

[22] C. B. Lucas, Atomic and Molecular Beams: Production and Collimation (CRC Press, Boca Raton, FL, 2013).

[23] D. R. Olander and V. Kruger, Molecular beam sources fabricated from multichannel arrays, III. The exit density problem, J. Appl. Phys. 41, 2769 (1970).

[24] H. Metcalf, Colloquium: Strong optical forces on atoms in multifrequency light, Rev. Mod. Phys. 89, 041001 (2017).

[25] P. R. Hemmer, M. Shahriar, H. Lamela-Rivera, S. P. Smith, B. Bernacki, and S. Ezekiel, Semiconductor laser excitation of Ramsey fringes by using a Raman transition in a cesium atomic beam, J. Opt. Soc. Am. B 10, 1326 (1993).

[26] T. L. Gustavson, P. Bouyer, and M. A. Kasevich, Precision Rotation Measurements with an Atom Interferometer Gyroscope, Phys. Rev. Lett. 78, 2046 (1997).
[27] K. Ross and B. Sonntag, High temperature metal atom beam sources, Rev. Sci. Instrum. 66, 4409 (1995).

[28] R. Senaratne, S. V. Rajagopal, Z. A. Geiger, K. M. Fujiwara, V. Lebedev, and D. M. Weld, Effusive atomic oven nozzle design using an aligned microcapillary array, Rev. Sci. Instrum. 86, 023105 (2015).

[29] M. Schioppo, N. Poli, M. Prevedelli, S. Falke, C. Lisdat, U. Sterr, and G. M. Tino, A compact and efficient strontium oven for laser-cooling experiments, Rev. Sci. Instrum. 83, 103101 (2012).

[30] P. Knapkiewicz, Alkali vapor MEMS cells technology toward high-vacuum self-pumping MEMS cell for atomic spectroscopy, Micromachines 9, 405 (2018).

[31] P. Knapkiewicz, Technological assessment of MEMS alkali vapor cells for atomic references, Micromachines 10, 25 (2019).

[32] L. V. Hau, J. Golovchenko, and M. M. Burns, A new atomic beam source: The "candlestick," Rev. Sci. Instrum. 65, 3746 (1994).

[33] M. Walkiewicz, P. Fox, and R. Scholten, Candlestick rubidium beam source, Rev. Sci. Instrum. 71, 3342 (2000).

[34] S. H. Wouters, G. ten Haaf, P. H. Mutsaers, and E. J. Vredenbregt, Design and experimental validation of a compact collimated Knudsen source, Rev. Sci. Instrum. 87, 083305 (2016).

[35] M. R. Walkiewicz, Manipulation of atoms using laser light, $\mathrm{Ph} . \mathrm{D}$. thesis, University of Melbourne, Department of Physics, 2000.

[36] R. E. Scholten (private communication).

[37] M. Partlow, X. Miao, J. Bochmann, M. Cashen, and H. Metcalf, Bichromatic Slowing and Collimation to Make an Intense Helium Beam, Phys. Rev. Lett. 93, 213004 (2004).

[38] C. Slowe, L. Vernac, and L. V. Hau, High flux source of cold rubidium atoms, Rev. Sci. Instrum. 76, 103101 (2005).

[39] W. Steckelmacher, The effect of cross-sectional shape on the molecular flow in long tubes, Vacuum 28, 269 (1978).

[40] MOLFLOW+ is a Monte Carlo code developed at CERN by R. Kersevan and M. Ady, website: cern.ch/molflow.

[41] D. Croucher and J. Clark, Total collision cross sections and van der Waals constants for alkali atom interactions with atoms and non-reactive diatomic molecules at thermal energies, J. Phys. B 2, 603 (1969).

[42] C. L. Yaws, Handbook of Vapor Pressure, Volume 4: Inorganic Compounds and Elements, (Gulf Professional Publishing, Houston, TX, 1995).

[43] M. Ady, Monte Carlo simulations of ultra high vacuum and synchrotron radiation for particle accelerators, Ph.D. thesis, Ecole Polytechnique Federale de Lausanne, 2016.

[44] M. D. Gregoire, Static polarizability measurements and inertial sensing with nanograting atom interferometry, Ph.D. thesis, The University of Arizona, 2016.

[45] C. I. Sukenik, M. G. Boshier, D. Cho, V. Sandoghdar, and E. A. Hinds, Measurement of the Casimir-Polder Force, Phys. Rev. Lett. 70, 560 (1993).

[46] X. Zheng, Y. R. Sun, J.-J. Chen, W. Jiang, K. Pachucki, and S.-M. Hu, Measurement of the Frequency of the $2{ }^{3} s-2{ }^{3} p$ Transition of ${ }^{4} \mathrm{He}$, Phys. Rev. Lett. 119, 263002 (2017).

[47] P. E. Gill, W. Murray, and M. H. Wright, Practical Optimization (Academic Press, London, 1981).

[48] P. C. Hansen, Regularization tools version 4.0 for MATLAB 7.3, Numerical Algorithms 46, 189 (2007). 
[49] D. A. Steck, Quantum and Atom Optics, available online at http://steck.us/teaching (revision 0.11.0, 18 Aug. 2016).

[50] N. Ramsey, Molecular Beams (Oxford University Press, Oxford, England, 1956), Vol. 20.

[51] H. Metcalf and P. van der Straten, Laser Cooling and Trapping (Springer, New York, 1999).

[52] J. Chen, Active optical clock, Chin. Sci. Bull. 54, 348 (2009).

[53] C. Brand, M. Sclafani, C. Knobloch, Y. Lilach, T. Juffmann, J. Kotakoski, C. Mangler, A. Winter, A. Turchanin, J. Meyer et al., An atomically thin matter-wave beamsplitter, Nat. Nanotechnol. 10, 845 (2015).

[54] S. Liron, D. G. Bopp, S. A. Schima, V. N. Maurice, and J. E. Kitching, Chip-scale atomic diffractive optical elements, Nat. Commun. 10, 3156 (2019).

[55] H. Alaeian, R. Ritter, M. Basic, R. Löw, and T. Pfau, Cavity QED based on room temperature atoms interacting with a photonic crystal cavity: A feasibility study, Appl. Phys. B 126, 1 (2020).

[56] A. Afanasiev, P. Melentiev, A. Kuzin, A. Y. Kalatskiy, and V. Balykin, Photon transport through a nanohole by a moving atom, New J. Phys. 18, 053015 (2016).

[57] N. Cooper, E. Da Ros, C. Briddon, V. Naniyil, M. Greenaway, and L. Hackermueller, Prospects for strongly coupled atomphoton quantum nodes, Sci. Rep. 9, 1 (2019).

[58] E. Da Ros, N. Cooper, J. Nute, and L. Hackermueller, Cold atoms in micromachined waveguides: A new platform for atomphoton interaction, arXiv:1906.06236.

[59] X. Zheng, Y. R. Sun, J.-J. Chen, J.-L. Wen, and S.-M. Hu, Lightforce-induced shift in laser spectroscopy of atomic helium, Phys. Rev. A 99, 032506 (2019).

[60] N. Sekiguchi, A. Hatakeyama, K. Okuma, and H. Usui, Scattering of an alkali-metal atomic beam on anti-spin-relaxation coatings, Phys. Rev. A 98, 042709 (2018).

[61] L. Sharma, A. Roy, S. Panja, and S. De, Atomic flux distribution from a low-divergent dark wall oven, Rev. Sci. Instrum. 90, 053202 (2019).

[62] D. Xiao, D. M. Lancaster, C. H. Allen, M. J. Taylor, T. A Lancaster, G. Shaw, N. R. Hutzler, and J. D. Weinstein, Shaped nozzles for cryogenic buffer-gas beam sources, Phys. Rev. A 99, 013603 (2019).

[63] Dr. Erol Kalkan, P. E., Deconvolution of two discrete time signals in frequency domain, MATLAB Central File Exchange (2020).

[64] D. A. Steck, Sodium D Line Data, available online https://steck. us/alkalidata (revision 2.2.1, 21 Nov. 2019). 